\title{
Minimally Invasive Plate Versus Intramedullary Interlocking Nailing In Distal Third Tibia Fractures
}

\author{
Deebak Kumar ${ }^{1}$, Ganesan Ganesan ram $^{2}$, Phagal Varthi Vijayaraghavan ${ }^{3}$ \\ 1, 2,3 (Department of orthopaedics, Sri Ramachandra Medical College/ University, India )
}

\begin{abstract}
Prospective study of sixty patients with distal tibia fracture who underwent surgical fixation at Sri Ramachandra University between June 2010 -June 2013. Of the 60 patients included in our study 30 underwent closed intramedullary interlocking nail and 30 were treated with plate osteosynthesis (MIPO). Patients were followed up for clinical and radiological evaluation using modified Klernn and Borner scoring system at 6 weeks, 3 months, 6 months, 1 year and 2 year. Plate osteosynthesis by minimally invasive technique and intramedullary interlocking nailing are equally effective methods of stabilisation for distal tibia fracture when considering the union rates and final functional outcome. However malunion, nonunion and secondary procedures were more frequent after intramedullary interlocking nail. Concurrent fibula fixation with plate osteosynthesis will minimise the incidence of malunion for distal tibia fractures. Randomized prospective evaluation of distal tibia fractures may clarify the efficacy of plate versus nail treatment and optimize the patient care.
\end{abstract}

Keywords: Distal third tibia, Intramedullary nail, mipo, malunion, malalignment

\section{INTRODUCTION}

Of all the long bones, the tibia and fibula have the highest incidence of fractures. These fractures are often caused by high energy trauma. The management of tibial fractures has always been particular interest to orthopaedic surgeons because they are relatively common but difficult to treat. The mode of treatment of distal third tibial fractures is still controversial. Of the available modalities of treatment, intramedullary interlocking nailing is one of the advocated ways of treating distal third tibial fractures. Biological plating Plate is also a preferred method of fixation for these distal third tibia fractures. In this study we are going to compare intramedullary Interlocking nailing and Minimally Invasive Plate Osteosynthesis for the management of distal third tibia fractures.

\section{AIM}

To compare the clinical and radiological outcome of extra-articular distal third tibia fractures treated by intramedullary interlocking nail and plate osteosynthesis (MIPO).

\section{MATERIALS AND METHODS}

Prospective study of sixty patients with distal tibia fracture who underwent surgical fixation at Sri Ramachandra University between June 2010 to June 2013. Of the 60 patients included in our study 30 underwent closed intramedullary interlocking nail and 30 were treated with plate osteosynthesis (MIPO). The inclusion criteria was closed extra - articular distal third tibia fractures (4 to $11 \mathrm{~cm}$ fro tibial plafond).The exclusion criteria were compound fractures, intra -articular fractures. Paediatric fractures, pathological fractures and any other associated fractures other than ipsilateral fibula. The age distribution ranged from 23 to 68 years with the mean age of 39.4 years. The mode of injury in all patients was due to vehicle accidents. Among the 60 patients included in our study there were 42 male and 18 female. The time of fixation varied from 6 hours to 18 days. All 60 patients were classified according to AO classification of which 30 belonged to A1, 28 belonged to A2, 2 belonged to A3. In the nailing group, 24 had ipsilateral tibia and fibula fracture and 6 patients had isolated tibia fracture. In the plating group there were 26 patients with ipsilateral tibia and fibula fracture and 4 patients had isolated tibia fracture.

All 60 patients were received in emergency room following which trauma series, relevant $\mathrm{x}$-rays including the affected legs with knee and ankle joints antero - posterior and lateral views were taken. Initial management consisted of closed reduction and splinting. All patients who underwent intramedullary interlocking nail were operated under regional anaesthesia with patient's supine on standard radiolucent table. Patellar splitting approach was used in all the patients. Reduction of fracture was achieved by manual traction using image intensifier. Nailing was done using standard technique and all fractures were fixed with two proximal and two distal locking screws.

Patients who underwent plate osteosynthesis by MIPO technique were operated under regional anaesthesia with patient supine on standard radiolucent table. By indirect fracture reduction technique, reduction 
of the fracture site was achieved and fixed with pre contoured plates and appropriate screws by the minimally invasive technique under the guidance of image intensifier. The decision for adjunctive fibular stabilisation as well as number of orientation of distal locking bolts was made at the surgeon's discretion. Duration of surgery and blood loss intra operatively was estimated for all 30 patients. None of the patients were operated under tourniquet control. Radiographic evaluation was done with standard antero -posterior and lateral view of the tibia with knee and ankle joint. Active range of movements of knee and ankle joint along with quadriceps strengthening exercises were started on the next day of surgery. All patients were given 3 to 5 days of broad spectrum intravenous antibiotics. Wound inspection was done on second, fifth, seventh post -operative day. Suture removal was done on 11 th post-operative day. Patients were maintained non or toe-touch followed by partial weight bearing until clinical and radiographic signs of healing were seen after which full weight bearing was allowed. Secondary surgeries like bone grafting, dynamization, implant exchange were performed as determined by the surgeon for failure of progression of healing, loss of fracture fixation or infection. Patients were followed up for clinical and radiological evaluation using modified Klernn and Borner scoring system at 6 weeks, 3 months, 6 months, 1 year and 2 year.

\section{RESULTS}

All the fractures united solidly with mean union time of 24.5 weeks ranging from 18 to 38 weeks. The patient who underwent nailing showed mean healing time of 24.9 weeks while patients treated with plate osteosynthesis by MIPO technique which is 24.2 weeks. Clinical and radiological outcome using modified Klemn and Borner scoring system in our study majority of patients had ended in excellent and good results. Patients who underwent nailing shows a good regain of range of movements in both knee and ankle. Two of the patients in plating group had ankle stiffness which compromised the overall functional outcome. In our series none of the patients had significant limb length discrepancy and no note of limping gait was made. Out of 60 patients with distal tibia fractures 50 patients were associated with distal fibula fractures. Fixation of fibula fractures usually results in better alignment. In plating group $90 \%$ of patients underwent fibula fixation. In this group mal alignment were resulted in 2 patients (antero posterior deformity). In nailing group only $40 \%$ of patients were treated with simultaneous fibula plating but the fractures were united in valgus /varus mal alignment in 6 cases. Even though fibula plating in nailing group will prevent malalignment sometimes it affects the fracture union rate and results in non union' s. In our series 4 patients in nailing group who had fibula fixation resulted in non union. These four patients had secondary procedures like implant exchange and bone grafting.

\section{DISCUSSION}

Distal tibial fractures which were treated with plate osteosynthesis especially after high energy injuries had encountered higher complication rates, because of which many surgeons preferred intramedullary interlocking nailing technique to minimise surgical insult to the fracture and adjacent soft tissue. However proximal and distal tibia fractures can he difficult to control with intramedullary device leading to malunion rates of 5-58\%. Minimally invasive plating technique reduces surgical trauma and maintain more biological environment for fracture healing ${ }^{1}$.Despite recently reported success of locking plates using minimally invasive plate osteosynthesis for distal tibia fracture the optimal treatment for these remain controversial. In our study, to analyse these two techniques various parameters like union rate, incidence of malalignment, rate of complications and functional outcome using Klemn and Borner scoring system were used. In the present study, average period of radiological union was found to be 24.2 weeks for the plating group and 24.9 weeks among the nailing group. In Tzeng et al study the mean union times were 22.6 weeks in nailing group and 27.8 weeks in the plating group. It has been found that preservation of soft tissue envelope and blood supply surrounding the fractures by using indirect reduction techniques would promote fracture healing regardless of type of fixation ${ }^{2}$.In our study 6 cases in the nailing group showed higher than acceptable range of angulation, malalignment or loss of reduction may occur after nailing mostly due to improper reduction, poor nail position (not centring the nail in the distal fragment), or loose fixation. Correct nail position, good contact of the nail to the end plate and rigid fixation of the nail-screw-bone construct should certainly be established ${ }^{3}$.

Among the plating group only two cases had significant rotational deformity. It has been found that indirect fracture reduction and fixation with medial plate was effective at restoring and maintaining alignment in high energy, mechanically unstable fractures of distal tibia. Malalignment has been recognised previously as a potential pitfall when using minimally invasive plating techniques because the bone is not directly visualised. Reports of $7 \%$ to $35 \%$ of distal tibia fractures treated with minimally invasive plate osteosynthesis have had problems with malalignment ${ }^{4}$ There were 3 cases of superficial infection in the nailing group, which healed on oral antibiotics. There was one case of infection among the plating group which required debridement with suturing and antibiotics. In both the groups spontaneous union occurred without any further complications. The mechanism resulting in distal tibial metaphyseal fractures usually consists of a high energy trauma that not only 
creates complicated fracture but also extends the injuries to the soft tissues.

This extended soft tissue injury could be further compromised by open reduction and internal fixation. Teeny et al, reported as many as 50 patients with atleast one major complications such as skin slough, wound dehiscence, infection, non-union. malunion or implant failure. Further more in case of recurrent osteomyelitis or soft tissue dehiscence, $16.6 \%$ of these patients eventually go on to amputation. Recently minimally invasive plating of injuries in this area has demonstrated less soft tissue problems and reasonably lessens the risk of infection ${ }^{4}$ It has been noted that concurrent fibula fixation among the patients treated by intramedullary interlocking nail will prevent malaligment but sometimes it affects the fracture union rate and results in nonunion $^{5}$. In our series 2 patients who were treated by intramedullary interlocking nail and concurrent fibula fixation resulted in non-union. Mosheiff et al and Schmidt et al recommended routine fixation of the concomitant fibular fractures to lessen the risk of malalignment. However, the essential benefit of intramedullary nailing in avoiding soft tissue dissection might be compromised in this way. No patient suffered from loss of reduction in thr series conducted by them demonstrating that it is not necessary to fix the fibula in the fractures of distal tibial metaphysis ${ }^{6}$.

It is possible that fibula fixation improves tibia alignment in some cases, but that an increased potential for delayed healing of the tibia may be seen when the fibula is stabilized concurrently. It has been noted angular malalignment of 5 degrees or more after nailing in $29 \%$ of patients, although malunion was not associated with presence of fibula fracture or fixation of fibula. Recent comparative stucy have also described more malalignment after nailing (50\% versus $17 \%$ after plating), suggesting that plating of complex fractures may result in less malalignment ${ }^{7}$. Recently, minimally invasive plating of injuries in this area has demonstrated excellent healing rates and only few required bone grafts. Sue et al, recently reviewed the literature and found 115 cases of minimally invasive plating for distal tibia fractures, and only $3 \%$ of these resulted in nonunion $^{8}$.After reviewing various articles, our study shows that plating by MIPO technique have given an equally good result when compared with the patients who had undergone intramedullary interlocking nail. In recent times distal tibia fractures treated by MIPO technique show minimal incidence of wound gaping and implant failure. These may be due to the evolution of minimally invasive surgical technique and better anatomical profile plates (thin profile and lock compression plates)

\section{VI. conclusion}

Plate osteosynthesis by minimally invasive technique and intramedullary interlocking nailing are equally effective methods of stabilisation for distal tibia fracture when considering the union rates and final functional outcome. However malunion, nonunion and secondary procedures were more frequent after intramedullary interlocking nail. Concurrent fibula fixation with plate osteosynthesis will minimise the incidence of malunion for distal tibia fractures. Randomised prospective evaluation of distal tibia fractures may clarify the efficacy of plate versus nail treatment and optimize the patient care.

\section{REFERENCES}

[1]. Resch H, Pechlaner S, Benedetto KP. Long term results after conservative and surgical treatment of fractures of the distal end of tibia.Aktuelle traumatol, 1986;16: 117-23.

[2]. Heather. A. Vallier MD, Toan Le MD. Radiographic and clinical comparison of distal tibia shaft fractures (4 to $11 \mathrm{cms}$ proximal to the tibial plafond) Plating vs Intramedullary nailing. J Orthop Trauma Vol 22, No.5, May/June 2008.

[3]. Cheng-yu Fan, Chao-Ching Chiang. Interlocking nails for displaced metaphyseal fractures of distal tibia. Injury 2005;36,669-674

[4]. Cory Collinge MD, Mark Kuper DO, Robert Protzman. Minimally invasiveplating of high energy Metaphyseal distal tibia fractures. J Orthop Trauma Vol 21, No.6, July 2007.

[5]. Cory Collinge MD, Robert Protzman MD. Outcome of minimally invasive plate osteosynthesis for metaphyseal distal tibia fractures. J Orthop Trauma. Vol 24, NO-1, Jan 2010.

[6]. Dorga AS, RuizAL, Thompson NS, Nolan Pc. Dia-metaphyseal distal tibial fractures -treatment with a shortened intramedullary nail: a review of 15 cases. Injury. 2000 dec;31 (10):799-804.

[7]. Egol KA, Weisz R, Hiebert R, Tejwani NC, Koval KJ, Sanders RW. Does fibular plating improve alignment after intramedullary nailing of distal tibia metaphyseal fractures. J Ortho Trauma;2006 Feb;20(2);94 - 103.

[8]. Gun-II Im and Sue-Kee Tae. Distal metaphyseal fractures of tibia: a prospective randomized trial of closed reduction and intramedullary nail versus open reduction and plate and screws fixation. J trauma 2005 nov; 59(5);1219-23. 